\title{
Research on topology identification technology of distribution station area based on distribution Transformer supervisory Terminal Unit
}

\author{
Min Zhang ${ }^{1,2}$, Qiang $\mathrm{Fu}^{1}$, Yu Xu${ }^{1}$, Hong Wei $\mathrm{Du}^{1}$ \\ ${ }^{1}$ NARI Technology Co., Ltd, Nanjing City, Jiangsu province, China \\ ${ }^{2}$ Nanjing Normal University, Nanjing City, Jiangsu province, China
}

\begin{abstract}
This paper proposes a topology identification idea based on the physical equipment and information fusion of the distribution network, which fully combines existing distribution automation master stations, intelligent distribution transformer terminals, smart meter equipment and information data resources, and uses carrier communication technology to reflect the topological relationship of the distribution station area, carry out research on key technologies of distribution network topology identification, in order to realize the comprehensive integration identification of the distribution station-linetransformer-household relationship and the distribution network topology structure, and improve data penetration between different systems ability to provide basic support for intelligent and lean operation and maintenance of the distribution network.
\end{abstract}

\section{Introduction}

In the distribution network system, the distribution station area refers to the power supply area from the distribution transformer to the user group, which is composed of the distribution transformer, the lowvoltage side feed line of the distribution transformer and the user group supplied by the distribution transformer. The distribution station area is the last "one kilometer" at the end of the distribution network and directly faces the end power users. Whether it can operate safely and economically directly affects the vital interests of the people and the operating costs and energy-saving goals of power companies. It affects the safety of electricity use for the broad masses of people. Therefore, the realization of safe, reliable, economical, and intelligent operation of distribution station areas has great social, engineering, and economic significance for the intelligent development of China's power grids.

For a long time, due to the inadequate planning of the low-voltage power distribution network construction, there is no uniform standard for the user's topology network in the station area. Part of the station network is not set up according to the rules, only for the purpose of powering on users, and lacks accurate topology information records. In addition, the original information of the user files is mostly in the form of paper cards, which are manually entered into the electronic information system one by one. There are cases where the entry errors cause the user's topological errors or omissions. In addition, during the operation of the distribution network station area, customers continuously increase and decrease adjustments. With the adjustment of the load cut-off network frame, the information changes are not timely, resulting in errors in user file updates, which are difficult to return.

The realization of the topology identification function of the distribution station area will effectively improve the economic operation level of the low-voltage distribution network, and clarify the relationship between the station area and the user, thereby improving the accuracy of the user file management of the power supply company and the refinement of the line loss management of the station area. It also clarifies the load planning of the area load, and provides detailed data for future power grid upgrades and new energy grid connection.

\section{Low-voltage household variable topology identification}

Please follow these instructions as carefully as possible so all articles within a conference have the same style to the title page. This paragraph follows a section title so it should not be indented[2-3].

\subsection{The principle of strategy}

The identification method of the low-voltage household variable topological relationship mainly relies on the carrier communication technology, making full use of the advantages of the carrier communication technology, that

zhangmin9@sgepri.sgcc.com.cn 
is, the carrier devices on the same power line can communicate with each other, so that the household variable topological relationship can be identified.

Taking the power distribution room in the community as an example, considering that the same-phase lines in the same station area are directly connected, except for a certain attenuation, the waveform is almost the same, so the phase line can be aligned at any position in the station area The voltage is modulated so that it is distorted, and the distortion can be detected in the same phase within the same station area, and even if the distortion signal becomes smaller, it can be reliably detected. When the voltage of a certain phase is distorted, because there is no loop with other phases, it will not affect other phases. Because the distribution transformer itself has a large inductance, it has a good suppression effect on this distortion. The distortion signal cannot be uploaded to the $10 \mathrm{KV}$ bus, so the distortion signal cannot be received by other transformers with the same bus, so the voltage distortion The signal is only valid at the current phase of the current station area. Based on the above principle, the voltage near the zero-crossing point is modulated so that the zero-crossing changes, so the receiving end only needs a zero-crossing detection circuit to achieve reliable identification. The modulation current will only exist between the modulation device and the transformer. The user only needs to detect the zerocrossing offset of the modulation voltage to communicate reliably. Therefore, after the device is powered on, the carrier in the TTU identifies all end devices in the station area through self-organization network, and the network is broadcasted. After receiving the network information, the end device returns its own device number, device type, and device type Mainly includes two kinds of branch ends and ends.

TTU receives the device number and device type sent by the terminal and records it. TTU can identify all devices at the terminal and the device type of the device. According to the device type, all the terminals are located in the line. The line can be completed by carrier communication recognition of the basic relationship of household changes.

Considering the situation of carrier communication crosstalk, there may be errors ( $95 \%$ accuracy rate) in the user-transformation relationship based on the carrier communication method. TTU can further verify the topological relationship based on the voltage similarity of the collected devices over a period of time.

When the local calibration is difficult, the TTU submits the non-conformance to the master station, and the master station performs the verification according to the global situation, and sends the verification result to the TTU terminal.

The above can complete the accurate identification of the relationship between households in the low-voltage station area.

\subsection{Identification process}

Taking the residential power distribution room shown in Figure. 1 as an example, the specific low-voltage household variable topology identification strategy process is as follows:

(i) After the TTU device in the station area is powered on (or the distribution automation master station issues a command to update the topology change to the TTU device), the carrier function of the TTU will automatically carry out the self-control of the equipment in the station area through the carrier communication broadcast method. The self-organizing network broadcast message will identify the branch box (branch box terminal) and the end meter (line end identification terminal) of all lines under this station area. After receiving the network information, the branch box terminal and the end identification terminal return its own device number ID and device type, where the device type includes two categories: branch end and end.

(ii) TTU receives the device number and device type of the branch box terminal and the terminal identification terminal and records it. According to the identified device type, TTU can classify the self-organized network equipment into branch terminal equipment and terminal equipment, and update and maintain the TTU. The topological relationship of households within the equipment; the above forms the basic topology file and uploads it to the main distribution automation station. 


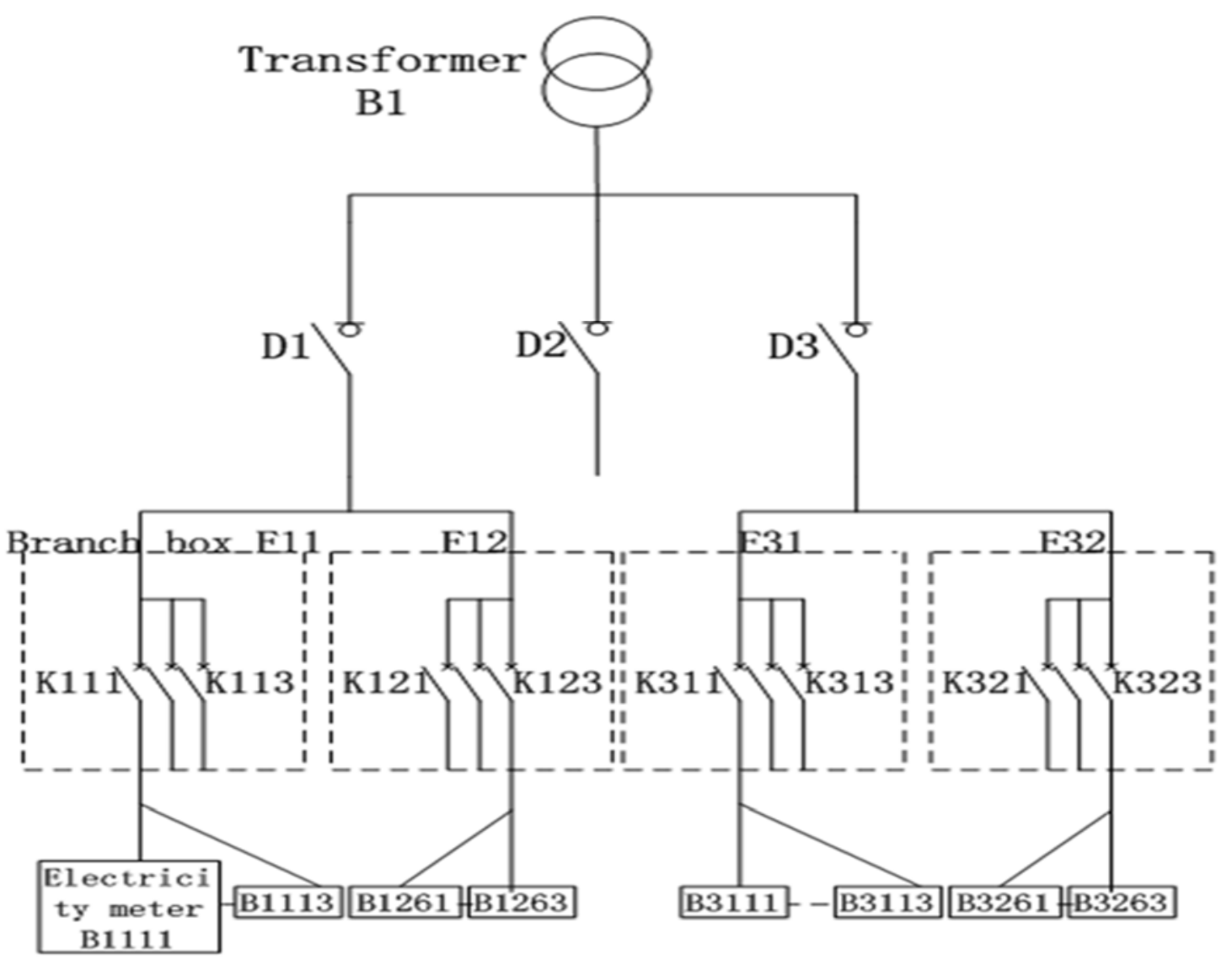

Figure 1. Schematic diagram of low-voltage topological relationship identification

(iii) TTU collects the voltage data sent by each device regularly, and judges the similarity of the voltage fluctuation of each device within 15 minutes. If there is any obvious abnormality, it can be considered that the device obviously does not belong to the station area, and it is excluded, and the result is notified to the host station.

(iv) According to the topology file and error information reported by each TTU, the master station summons the corresponding TTU (the same station TTU) to re-network to eliminate the fault, and finally realize the accurate identification of the topology change relationship.

\section{Identification of low-VOLTAGE hierarchical relationships}

\subsection{The principle of strategy}

The identification of the low-voltage topology hierarchy relationship is based on the method of generating characteristic current signals at the end of the line. TTU controls the end module on the line to generate the characteristic current signal, thereby changing the waveform characteristics of the current on the line. The hierarchical branch box node module will collect the characteristic current signal and report it to the TTU. The TTU records the branch box node module topology information corresponding to the end module and sends it to the distribution automation master station in time by the TTU, and so on to complete the hierarchical relationship of the entire line Recognition. The topology flowchart is shown in Figure 2 


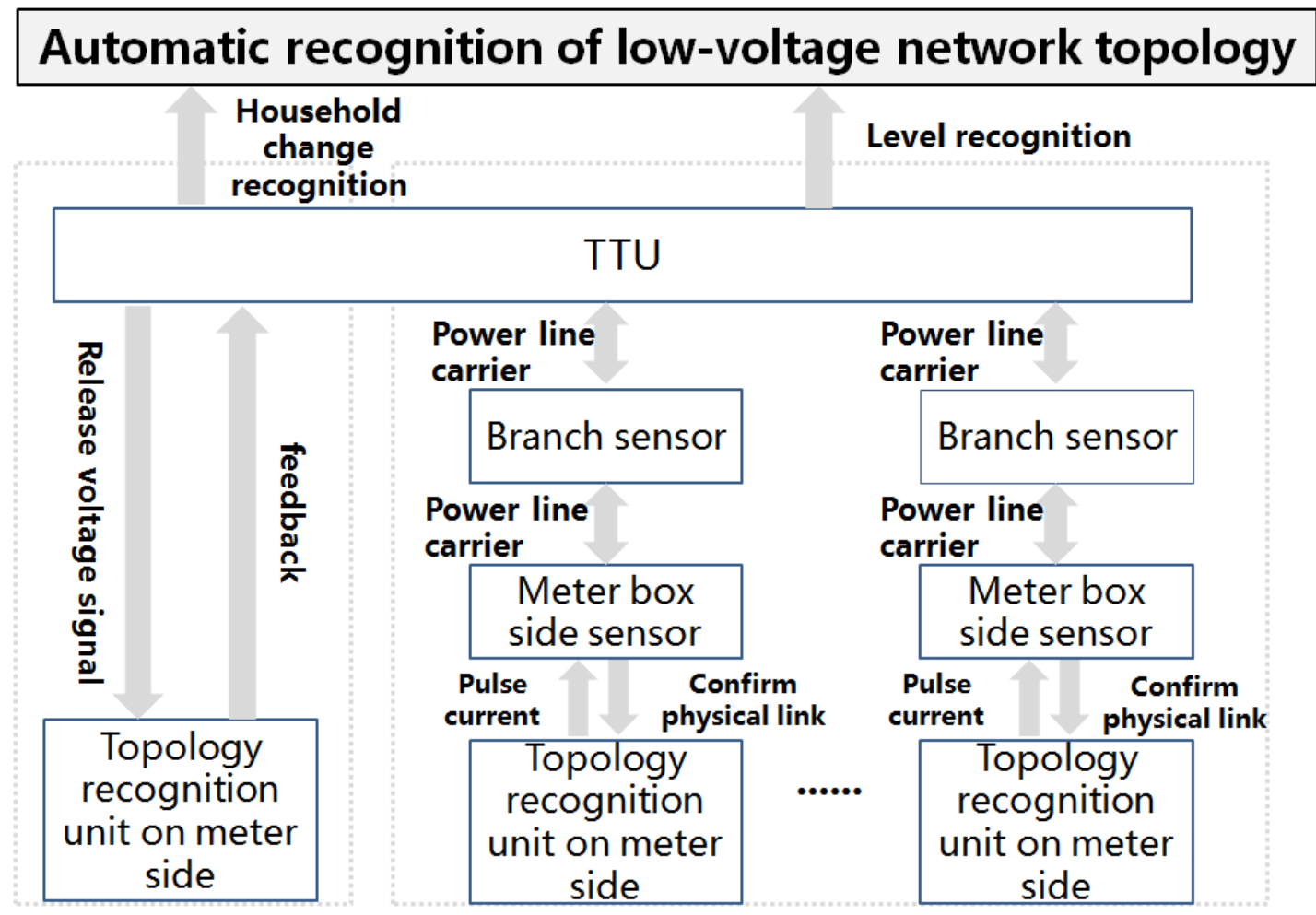

Figure 2. Flow chart of topology identification

\subsection{Identification process}

Continuing to take the community distribution room shown in Figure. 1 as an example, the specific lowvoltage hierarchical relationship identification strategy process is as follows:

(i) TTU collects line voltage and current in real time. In order to ensure the test accuracy of small signal current, TTU separately controls the terminal identification unit to send characteristic current signals when the line current is minimum.

(ii) The branch box module on the line records the characteristic current signal and records and returns the SOE information to the TTU. The TTU will update and record the branch box node corresponding to the end identification unit. Take the B1111 in front of the table shown in Fig. 1 as an example. The terminal identification unit of B1111 in front of the control table sends the characteristic current signal, the K111 branch module in the corresponding branch box receives the corresponding characteristic current signal, and then the K111 branch module sends SOE information to TTU, so that the TTU updates and records the topology of the line
B1-K111-B1111, and so on to identify all topological structures.

(iii) Finally, TTU will send the identified hierarchy relationship to the distribution automation master station in time. After receiving the information sent by TTU, the distribution automation main station forms a complete and accurate topology relationship of the station area according to the power supply path of each end unit and the hierarchy of the station area.

\section{Applications}

According to the identification method mentioned above, the corresponding intelligent terminal and sensing equipment are adopted in a certain station in Nanjing. After the above process, the distribution terminal forms a topology file and uploads it to the distribution network master station. The main station displays the hierarchical structure and equipment list of the station area-branch line-meter box in a tree diagram, and compares the data of the main station low voltage model with the PMS2.0 low voltage ledger data in the form of a table. 


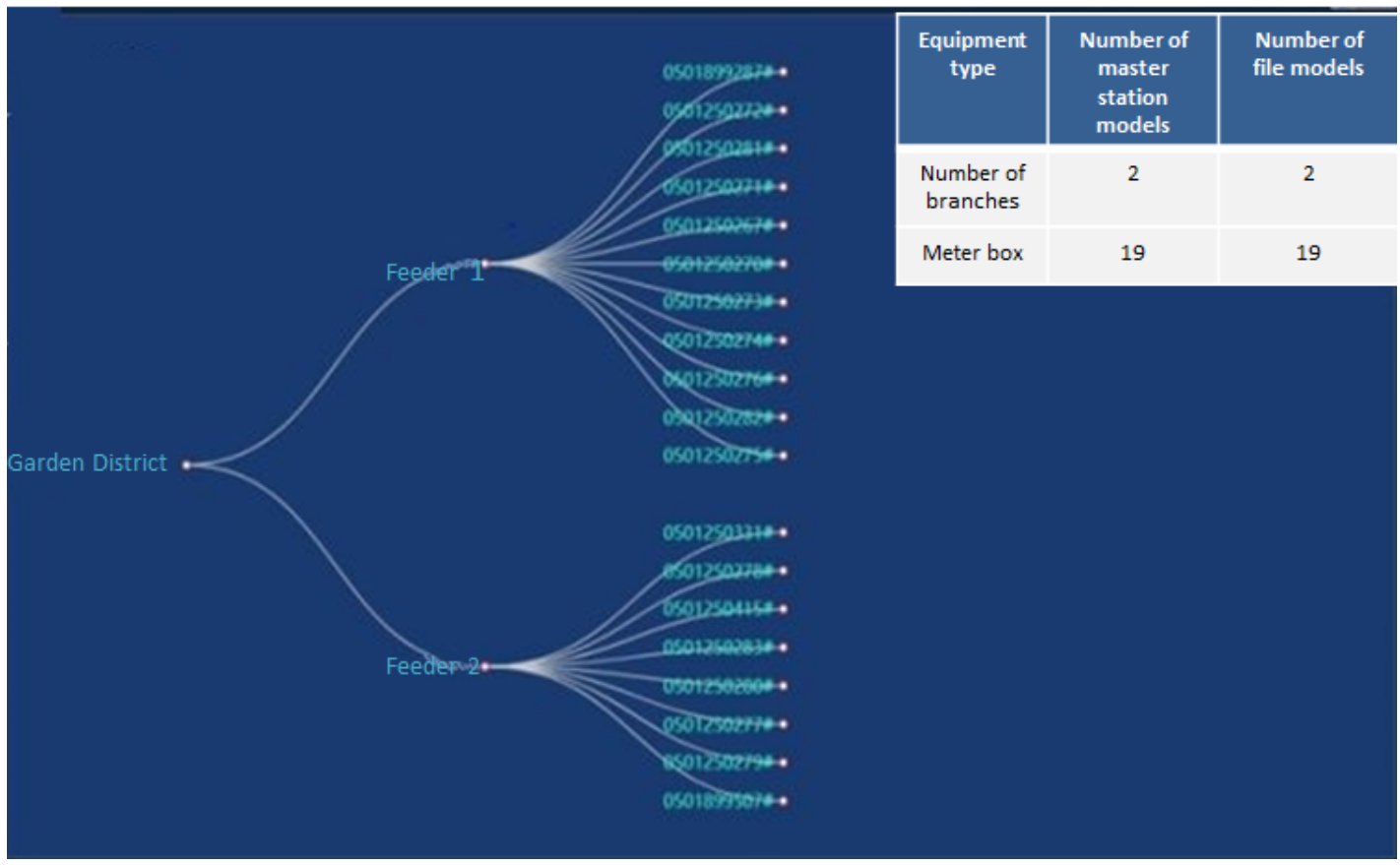

Figure 3. Topology diagram of distribution master station

\section{Conclusion}

This paper presents a research on topology recognition technology. Relying on the intelligent distribution transformer terminal in the distribution station area, the intelligent sensing equipment with signal detection devices installed at the branch box and meter box can automatically identify the hierarchical relationship of households and form a topology file. Through the automatic identification technology of distribution network topology, it provides technical support means for the automatic detection, analysis and generation of topology of distribution network areas, hoping to effectively solve the problem of low accuracy of lowvoltage distribution network topology, and help improve lean management level of distribution network, such as transportation and inspection, marketing, regulation and control.

\section{References}

1. Jian Liu, Shuren Zhao, Xiaoqing Zhang. (2012) Progress and some suggestions of distribution automation in China [J]. Power System Automation, 36 (19): 6-10+21.

2. Bingbing Shen, Lin $\mathrm{Wu}$, Peng Wang. (2012) Technical characteristics and application effectiveness analysis of the pilot project of distribution automation [J]. Power System Automation, 36 (18): 27-32.

3. Lei Wei, Weiwei Miao, Xinjian Zhao. (2014) Selection and construction plan of distribution automation communication technology [J]. Power Supply and Use, (5): 38-42.
4. Xu Ma, Fang Yang et al. (2015) Network topology reconstruction technology based on power line communication $[\mathrm{J}]$. Telecommunications Science, 31 (12): 46-51.

5. Mingwei Li, Chuanyuan Zhang, Xiaokun Ma, Wei Liang. (2018) Research on automatic identification technology of network topology and phase difference in low-voltage distribution station area $[\mathrm{J}]$. Electric Age, (10): 62-63.

6. Geng Juncheng, Zhang Xiaofei, Guo Zhimin, et al. (2017) Low-voltage distribution network topology verification method based on discrete Fréchet distance and clipping nearest neighbor method[J]. Electrical Measurement and Instrumentation, 54(5): 50- 55.

7. Pan Mingming, Tian Shiming, $\mathrm{Wu}$ Bo, et al. (2017) Research on station area identification and electricity theft detection method based on smart meter data[J]. Smart Electric Power, 45(12):80-84.

8. Aliaosha. Ye, Gu Jun, Zhang Xiaoqiu (2018) Intelligent station area recognition technology based on data spatio-temporal correlation[J]. Modern Electronic Technology, 41(04):30-33.

9. Li Ya, Fan Rusen, Jiang Wei, et al. (2017) Research on intelligent station area recognition method based on BP neural network [J]. Electrical Measurement and Instrumentation, 2017, 54(3): 25-30

10. KAS N, TOR O B,, CEBECI M E, et al. (2017) Theoreticak and practical aspects of implementing a low voltage express feeder between nearby distribution transformers to reduce annual losses [C] Smart Grid and Cities Congress and Fair. Istanbul: IEEE,70-74 